\title{
Localization of 3D Anatomical Point Landmarks in 3D Tomographic Images Using Deformable Models ${ }^{\star}$
}

\author{
Sönke Frantz, Karl Rohr, and H. Siegfried Stiehl \\ Universität Hamburg, Fachbereich Informatik, Arbeitsbereich Kognitive Systeme \\ Vogt-Kölln-Str. 30, 22527 Hamburg, Germany \\ \{frantz,rohr, stiehl\}@informatik. uni-hamburg.de \\ http://kogs-www . informatik. uni-hamburg. de/PROJECTS/imagine/Imagine.html
}

\begin{abstract}
Existing differential approaches to the localization of $3 \mathrm{D}$ anatomical point landmarks in 3D tomographic images are relatively sensitive to noise as well as to small intensity variations, both of which result in false detections as well as affect the localization accuracy. In this paper, we introduce a new approach to 3D landmark localization based on deformable models, which takes into account more global image information in comparison to differential approaches. To model the surface at a landmark, we use quadric surfaces combined with global deformations. The models are fitted to the image data by optimizing an edge-based fitting measure that incorporates the strength as well as the direction of the intensity variations. Initial values for the model parameters are determined by a semi-automatic differential approach. We obtain accurate estimates of the 3D landmark positions directly from the fitted model parameters. Experimental results of applying our new approach to 3D tomographic images of the human head are presented. In comparison to a pure differential approach to landmark localization, the localization accuracy is significantly improved and also the number of false detections is reduced.
\end{abstract}

\section{Introduction}

In this contribution, we address the problem of extracting 3D anatomical point landmarks from 3D tomographic images, focusing on anatomical structures of the human head. The driving task is landmark-based 3D image registration, which is fundamental to computer-assisted neurosurgery. Existing work on the automated extraction of 3D point landmarks is based on differential approaches (e.g., [17],[11]). However, while being computationally efficient, differential approaches are relatively sensitive to noise as well as to small intensity variations, both of which result in false detections as well as affect the localization accuracy. In this paper, we introduce a new approach to 3D landmark localization based on

\footnotetext{
* This work was supported by Philips Research Hamburg, project IMAGINE (IMageand Atlas-Guided Interventions in NEurosurgery).
} 
deformable models, which takes into account more global image information in comparison to differential approaches and thus opens the possibility of increasing the robustness as well as the accuracy in landmark extraction. Previously, deformable models have been applied to segmentation, tracking, and image registration (see [8] for a survey), whereas the localization of 3D point landmarks based on deformable models has not been considered so far.

Exemplarily, we here focus on two different types of landmarks, namely, salient surface loci (curvature extrema) of tips and saddle structures. Examples of corresponding 3D point landmarks of the human head are the tips of the ventricular horns or the saddle points at the zygomatic bones (see Fig. 1). To represent such structures, we utilize 3D surface models. In the literature, a variety of 3D surface models has been used (e.g., [15],[13],[3],[14],,[7],[16],,[19],[1]). Note that in comparison to earlier work on deformable models, we are here interested in the accurate localization of salient surface loci. Central to an efficient solution of this specific problem is that the model surface exhibits a unique point whose position can be directly computed from the model parameters. As a compromise between generality and efficiency, we here use quadric surfaces as shape prototypes, which are combined with additional global deformations to enlarge the range of shapes (Sect. 2).
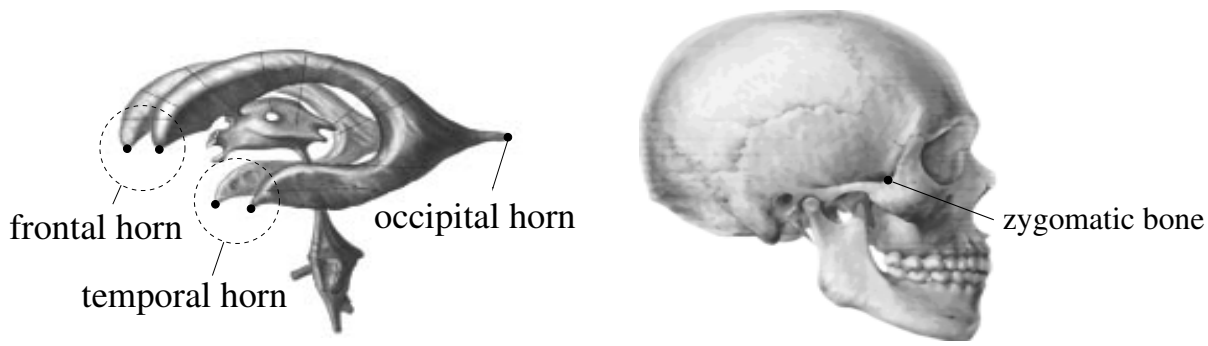

Fig. 1. Ventricular horns of the human brain (from [12]) and the human skull (from [2]). Examples of 3D point landmarks are indicated by black dots.

Model fitting is formulated as an optimization problem where a suitable fitting measure is optimized w.r.t. the model parameters. Here, we use an edgebased fitting measure that incorporates the strength as well as the direction of the intensity variations. This measure, which is described in Sect. 3, is a $3 \mathrm{D}$ generalization of a $2 \mathrm{D}$ fitting measure suggested in [20]. Usually, only the strength of the intensity variations is incorporated (e.g., [19],[14]). To determine initial values for the model parameters, we have developed a semi-automatic differential approach (Sect. 4). Experimental results of applying our new approach to 3D tomographic images of the human head are presented in Sect. 5. In particular, we analyze the localization accuracy of our new approach and compare it with that of an existing differential approach. 


\section{Geometric Models of Tips and Saddle Structures}

As 3D shape prototypes, we here use quadric surfaces, namely, ellipsoids for 3D tip-like structures and hyperboloids of one sheet for 3D saddle structures. However, real structures in general show deviations from these prototypes (e.g., the ventricular horns generally have a bended shape and partly show a tapering). To take into account bending in the case of tip-like structures, we here additionally apply a quadratic bending deformation along the centerline of the ellipsoid (i.e., the $z$-axis) [3]: $\mathcal{B}(\mathbf{x})=\left(x+\delta \cos v z^{2}, y+\delta \sin v z^{2}, z\right)^{T}$, where $\mathbf{x}=(x, y, z)^{T}$ denotes an arbitrary surface point and $\delta \geq 0$ determines the strength and $v$ the direction of bending. To transform the object-centered model coordinate system to the image coordinate system, we here use a rigid transformation, $\mathcal{R}(\mathbf{x})=\mathbf{R x}+\mathbf{t}$, where $\mathbf{t}=(X, Y, Z)^{T}$ denotes the translation vector and $\mathbf{R}$ the rotation matrix depending on the rotation angles $\alpha, \beta, \gamma$.

Tips. The parametric form of our model is obtained by applying the bending deformation and the rigid transformation to the parametric form of an ellipsoid:

$$
\boldsymbol{x}_{t i p}(\theta, \phi)=\mathbf{R}\left(\begin{array}{l}
a_{1} \cos \theta \cos \phi+\delta \cos v\left(a_{3} \sin \theta\right)^{2} \\
a_{2} \cos \theta \sin \phi+\delta \sin v\left(a_{3} \sin \theta\right)^{2} \\
a_{3} \sin \theta
\end{array}\right)+\mathbf{t},
$$

where $0 \leq \theta \leq \pi / 2$ and $-\pi \leq \phi<\pi$ are the latitude and longitude angle parameters, resp., and $a_{1}, a_{2}, a_{3}>0$ are scaling parameters. Hence, the model is described by the parameter vector $\mathbf{p}=\left(a_{1}, a_{2}, a_{3}, \delta, v, X, Y, Z, \alpha, \beta, \gamma\right)$. The landmark position of our model, i.e., the position of the curvature extremum of the deformed ellipsoid, is given by $\mathbf{x}_{l}=\boldsymbol{x}_{t i p}(\pi / 2,0)=\mathbf{R}\left(\delta \cos v a_{3}^{2}, \delta \sin v a_{3}^{2}, a_{3}\right)^{T}+\mathbf{t}$. Figure 2 (left) shows an example of a bended tip-like structure.

Saddle structures. Here, the parametric form of our model is obtained by applying the rigid transformation to the parametric form of a hyperboloid of one sheet:

$$
\boldsymbol{x}_{\text {saddle }}(\theta, \phi)=\mathbf{R}\left(\begin{array}{l}
a_{1} \sec \theta \cos \phi \\
a_{2} \sec \theta \sin \phi \\
a_{3} \tan \theta
\end{array}\right)+\mathbf{t},
$$

where $|\theta|<\pi / 2$ and $0 \leq \phi \leq \pi$. Thus, the model is described by the parameter vector $\mathbf{p}=\left(a_{1}, a_{2}, a_{3}, X, Y, Z, \alpha, \beta, \gamma\right)$. The landmark position is here given by $\mathbf{x}_{l}=\boldsymbol{x}_{\text {saddle }}(0, \pi / 2)=\mathbf{R}\left(0, a_{2}, 0\right)^{T}+\mathbf{t}$. Figure 2 (right) shows an example of a saddle structure.

\section{Model Fitting Using an Edge-Based Fitting Measure}

The geometric models introduced in Sect. 2 are fitted to the image data by optimizing an edge-based fitting measure w.r.t. the model parameters. Our fitting measure, which is a generalization of the $2 \mathrm{D}$ fitting measure in [20], exploits (a) the similarity between the directions of the intensity gradient and the normals of the model surface as well as (b) the strength of the intensity variations. 

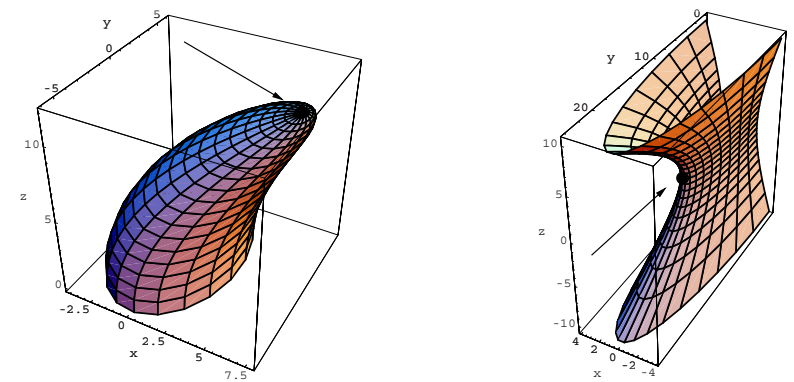

Fig. 2. Geometric models based on quadric surfaces as $3 \mathrm{D}$ shape prototypes. The landmark positions are indicated by a black dot.

As a result, the influence of neighboring structures during fitting is diminished significantly, which increases the robustness of model fitting.

During fitting, we consider the contributions of the intensity gradient in the direction of the normal of the model surface, utilizing the projection of the intensity gradient onto the unit normal of the model surface:

$$
M_{f i t}(\mathbf{p})= \pm \iint<\nabla g(\boldsymbol{x}(\theta, \phi ; \mathbf{p})), \frac{\partial \boldsymbol{x}(\theta, \phi ; \mathbf{p})}{\partial \theta} \times \frac{\partial \boldsymbol{x}(\theta, \phi ; \mathbf{p})}{\partial \phi}>d \theta d \phi \rightarrow \text { min. }
$$

where $g$ denotes the intensity function, $\boldsymbol{x}$ is the parametric form of the respective geometric model, which depends on $\theta, \phi$, and the model parameter vector $\mathbf{p}$, and $<\cdot, \cdot>$ denotes the inner product. The choice of the sign \pm of the fitting measure depends on the appearance of the landmark at hand in the image: In the case of a dark structure, where the intensity gradient points outward, the sign is positive, whereas the sign is negative in the case of a bright structure. Thus, the better the similarity between the directions of the intensity gradients and the normals of the model surface is and the stronger the intensity variations along the model surface are, the smaller is the measure in Eq. (3). It is worth noting that in our implementation, only those surface points in Eq. (3) are considered where $\pm<\nabla g(\boldsymbol{x}(\theta, \phi ; \mathbf{p})), \frac{\partial \boldsymbol{x}(\theta, \phi ; \mathbf{p})}{\partial \theta} \times \frac{\partial \boldsymbol{x}(\theta, \phi ; \mathbf{p})}{\partial \phi}>$ is less than zero. That is, surface points where the direction of the intensity gradient differs significantly from the surface normal are excluded. As a result, the influence of neighboring structures is further reduced. We optimize the fitting measure in Eq. (3) w.r.t. the model parameters by applying the conjugate gradient method ([10]).

A similar 3D fitting measure was suggested in [5]. However, in [5] a discrete formulation of Eq. (3) was used. Also, different geometric models based on Fourier surfaces were used in [5], and the approach was applied to segmented data only, while here we do not require segmented data.

\section{Initialization of the Model Parameters}

A central issue of fitting deformable models to the image data is the determination of suitable initial values for the model parameters. Often, initial values are 
manually determined, which is tedious and time-consuming. Here, we initialize the model parameters using a semi-automatic procedure. The model for tip-like structures is initialized using an ellipsoid as approximation. Thus, for both models from Sect. 2, we have to find initial values for nine parameters (translation, rotation, and scaling).

An initial estimate of the landmark position, $\hat{\mathbf{x}}_{l}$, is obtained by a semiautomatic differential approach ([11],[6]). To initialize the rotation angles $\alpha, \beta, \gamma$, we exploit the pose of the local isointensity surface at the estimated landmark position, assuming that the local isointensity surface, which is defined by the implicit equation $g(\mathbf{x})-g\left(\hat{\mathbf{x}}_{l}\right)=0$, well approximates the surface of the anatomical structure at hand. The rotation angles are then determined by the direction of the intensity gradient $\nabla g\left(\hat{\mathbf{x}}_{l}\right)$ (estimate of the normal) as well as the principal curvature directions of the local isointensity surface at $\hat{\mathbf{x}}_{l}$ (see, e.g., [4],[17] for computing the curvature of isointensity surfaces). The scaling parameters $a_{1}, a_{2}, a_{3}$ are initialized based on the principal curvatures $\kappa_{1}, \kappa_{2}$ of the local isointensity surface at the estimated landmark position. For example, in the case of a tip, we have the relations $\kappa_{1}=a_{3} / a_{1}^{2}$ and $\kappa_{2}=a_{3} / a_{2}^{2}$. Note, however, that we have only two principal curvatures, while we have three scaling parameters. To cope with this problem, we here initialize one scaling parameter manually.

\section{Experimental Results for 3D Tomographic Images}

We have applied our approach to 3D synthetic data as well as to 3D tomographic images of the human head. In this section, we present experimental results of applying our approach to a 3D MR image and a 3D CT image of one patient. In Sect. 5.1, we describe the parameter setting, and in Sect. 5.2 we present the results obtained for different anatomical landmarks of the human head.

\subsection{Parameter Setting}

In the case of the $3 \mathrm{D}$ MR image (T1-weighting, voxel size $\approx 0.86 \times 0.86 \times 1.2 \mathrm{~mm}^{3}$ ), we considered the tips of the frontal and occipital ventricular horns as well as the saddle points at the zygomatic bones (see Fig. 1, where the landmarks are indicated). The field-of-view of the CT image captures only a part of the ventricular horns, and therefore we here considered only the zygomatic bones. Instead of the original, anisotropic CT data, we used interpolated data with an isotropic voxel size of $1.0^{3} \mathrm{~mm}^{3}$ (for interpolation we applied the approach in [9]).

Initial estimates of the landmark positions were determined by applying the semi-automatic differential approach in [11],[6], where partial derivatives of the intensity function were estimated using cubic B-spline image interpolation ([18]) and Gaussian smoothing. The scale of the Gaussian filters was coarsely adapted to the scale of the respective landmark: For the ventricular horns we used $\sigma=1.5 \mathrm{~mm}$, while for the zygomatic bones we used $\sigma=1.0 \mathrm{~mm}$ (in our implementation of derivative computation, the filter scales are specified in units 
of $\mathrm{mm}$ and are afterwards converted to voxel units based on the respective image resolution). In case of several detections, we selected the candidate with the maximal operator response. For computing the curvature of the local isointensity surface, the scale of the Gaussian filters was the same as that used for landmark detection.

The fitting measure $M_{f i t}(\mathbf{p})$ as well as its derivative w.r.t. the model parameter vector $\mathbf{p}\left(\nabla M_{f i t}(\mathbf{p})\right.$ is required for optimization) involve (a) the parametric forms of the models as well as partial derivatives of the parametric forms w.r.t. $\theta, \phi$, and the model parameters and (b) image derivatives. Expressions involving the parametric forms were determined analytically. Image derivatives were computed using Gaussian filters with $\sigma=1.0 \mathrm{~mm}$. For numerical evaluation of the integral in the fitting measure in Eq. (3) and in its derivative, we adopted a scheme based on equidistant sampling of the two-dimensional parameter space $(\theta, \phi)$ and cubic interpolation $([10])$. The image derivatives were trilinearly interpolated. To diminish the influence of neighboring structures, model fitting was restricted to a spherical region-of-interest (ROI) centered at the estimated landmark position, where the ROI radius was set to 15 voxels.

\subsection{Results for the Ventricular Horns and the Zygomatic Bones}

Ventricular Horns We considered four different landmarks, namely, the tips of the frontal and occipital ventricular horns in both hemispheres. For each landmark, the semi-automatic differential approach in [11],[6] yielded a reasonable initial estimate of the landmark position. The rotation angles $\alpha, \beta, \gamma$ as well as the scaling parameters $a_{1}$ and $a_{2}$ were automatically determined based on the differential characteristics of the local isointensity surfaces at the position estimates (see Sect. 4). Only the scaling parameter $a_{3}$ was manually initialized. The bending parameters were initially set to zero. Figure $3 \mathrm{a}$ visualizes the initialization result obtained for the left frontal ventricular horn; for the other landmarks, we obtained similar initialization results.

Given the relatively large number of parameters, model fitting was then performed in two steps for reasons of robustness: To achieve a coarse adaption, we first fitted only the six parameters of the rigid transformation, while the other parameters were kept constant. In the second step, all parameters (translation, rotation, scaling, and bending) were considered during optimization. Model fitting took in total between 34 and 184 seconds (SUN SPARC Ultra 2) and succeeded in all cases. Figure $3 \mathrm{~b}$ exemplarily shows the fitting result obtained for the left frontal ventricular horn. One can see that the fitted model surface well agrees with the ventricle surface.

The localized landmark positions derived from the fitted model turned out to be good. Figures 3c and 4 show the localization results obtained for the tips of the left frontal and occipital horn, resp. Please note that for visualization purposes, the model surfaces as well as the landmark positions are represented by voxel positions only, while the fitting results yield subvoxel positions. 

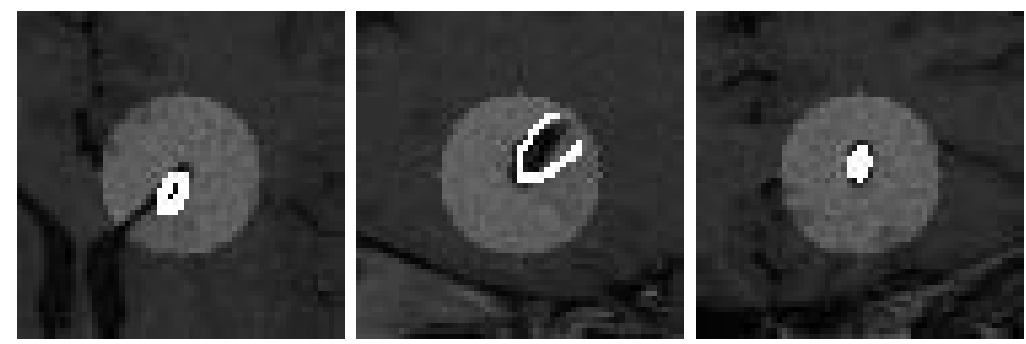

(a) Model initialization
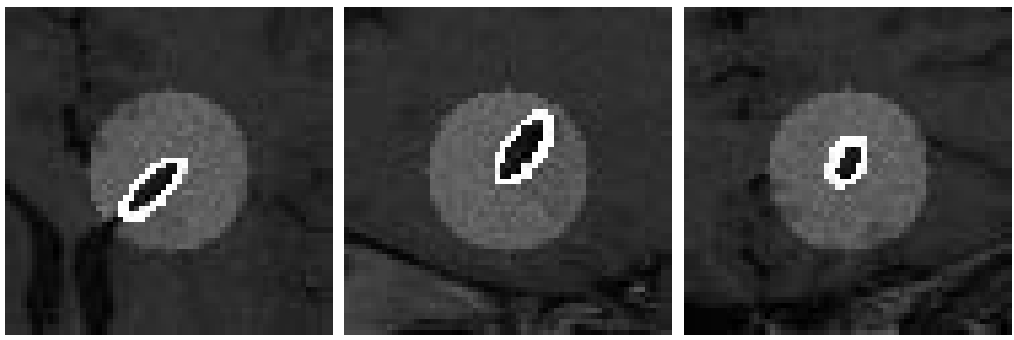

(b) Fitting result
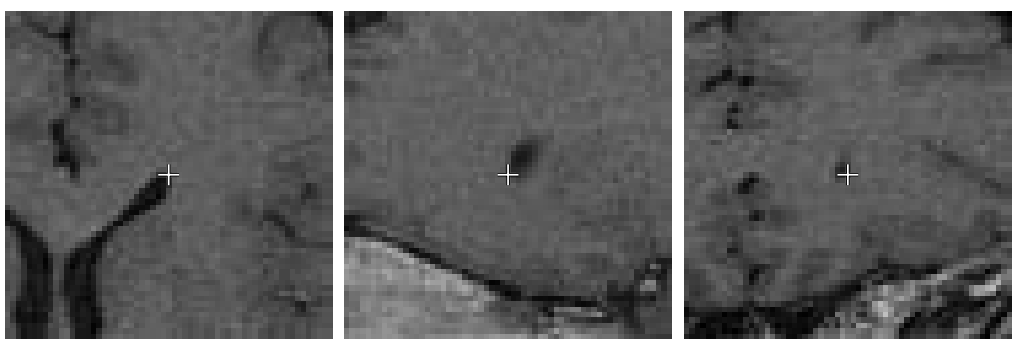

(c) Localized landmark position

Fig. 3. Localization of the tip of the left frontal ventricular horn in a 3D MR image. Orthogonal sections at the ROI center depicting (a) the surface initialization and (b) the fitting result. The considered spherical ROI is highlighted. (c) Orthogonal sections at the localized landmark position based on the fitted model (white cross).
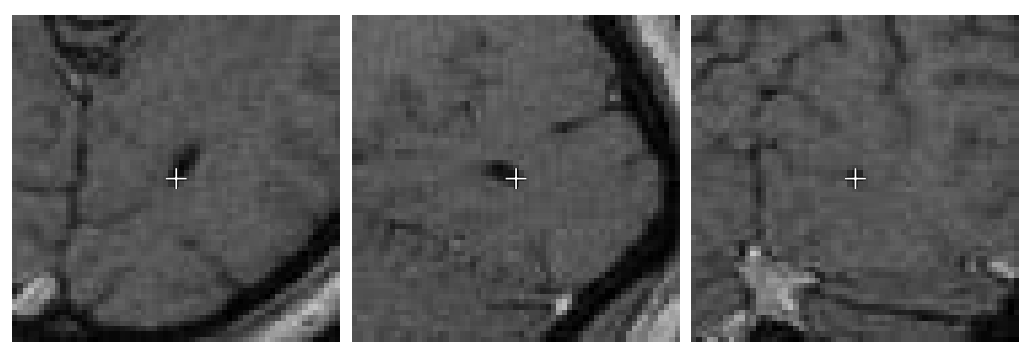

Fig. 4. Localization of the tip of the left occ. ventr. horn in a 3D MR image. Orthogonal sections at the localized landmark position based on the fitted model (white cross). 
Zygomatic Bones In both modalities, we obtained reasonable initial values for the model parameters. Only the scaling parameters were manually coarsely initialized. In contrast to the experiments using the ventricular horns, we here performed model fitting in a single step in which all parameters were adapted. Model fitting took between 35 and 117 seconds and gave in all cases good results. The localized landmark positions derived from the fitting results are in all cases satisfying as visual inspection revealed. Figure 5 shows the localization results for the saddle point at the left zygomatic bone in MR and CT.
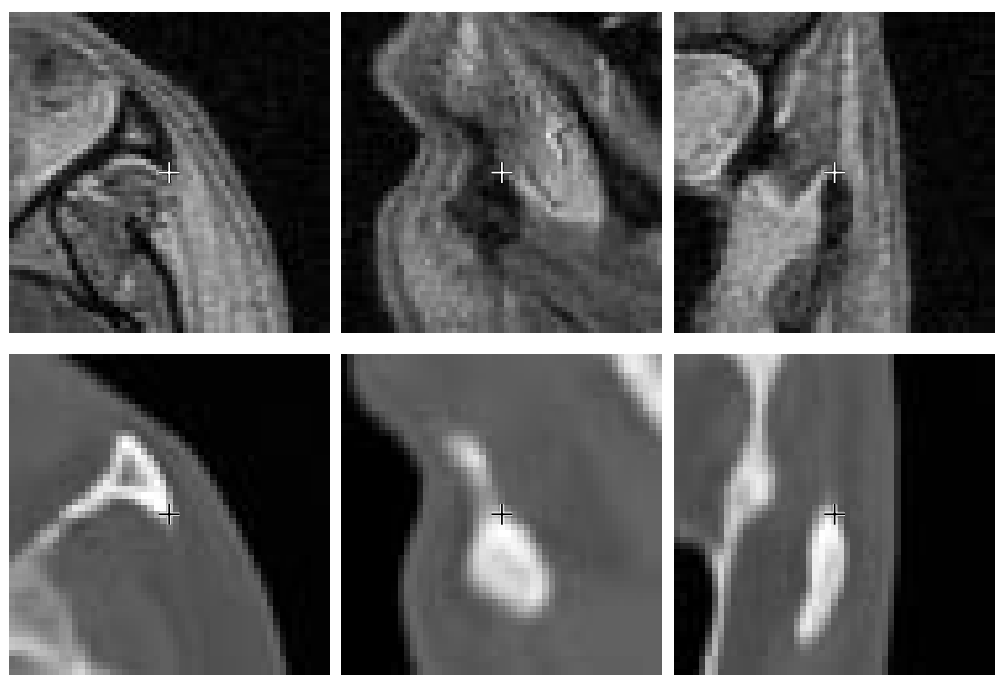

Fig. 5. Localization of the saddle point at the left zygomatic bone in a 3D MR image (top) and a 3D CT image (bottom). Orthogonal sections at the localized landmark position based on the fitted model (white (black) cross in the MR (CT) image).

Localization Accuracy We now analyze the localization accuracy of our new approach in the case of the MR image, using as ground truth positions that were manually determined in agreement with up to four persons. Note that manually only voxel positions were determined, while our new approach yields subvoxel positions. For comparison, we use the results obtained with a differential approach $([11],[6])$, which was here also used to determine initial estimates of the landmark positions. Table 1 summarizes the computed Euclidean distances to the ground truth positions for six landmarks. One can see that for each landmark, the locus obtained with our new approach based on deformable models is better (i.e., closer to the reference position) than the locus obtained with the differential approach. The mean Euclidean distance from the positions localized with our new approach to the reference positions is $\bar{e}_{\text {new }}=1.22 \mathrm{~mm}$, while for the differential approach we have $\bar{e}_{\text {differential }}=2.11 \mathrm{~mm}$. Thus, the localization accuracy was improved by $0.89 \mathrm{~mm}$. 


\begin{tabular}{|l|c|c|}
\cline { 2 - 3 } \multicolumn{1}{c|}{} & $e_{\text {differential }}$ & $e_{\text {new }}$ \\
\hline Left frontal horn & $1.92 \mathrm{~mm}$ & $0.90 \mathrm{~mm}$ \\
\hline Left occipital horn & $3.32 \mathrm{~mm}$ & $1.23 \mathrm{~mm}$ \\
\hline Left zygomatic bone & $0.86 \mathrm{~mm}$ & $0.78 \mathrm{~mm}$ \\
\hline
\end{tabular}

\begin{tabular}{|l|c|c|}
\cline { 2 - 3 } \multicolumn{1}{c|}{} & $e_{\text {differential }}$ & $e_{\text {new }}$ \\
\hline Right frontal horn & $1.72 \mathrm{~mm}$ & $1.28 \mathrm{~mm}$ \\
\hline Right occipital horn & $2.58 \mathrm{~mm}$ & $1.61 \mathrm{~mm}$ \\
\hline Right zygomatic bone & $2.26 \mathrm{~mm}$ & $1.52 \mathrm{~mm}$ \\
\hline
\end{tabular}

Table 1. Comparison of the localization accuracy of a differential approach to landmark localization $\left(e_{\text {differential }}\right)$ and our new approach based on deformable models $\left(e_{\text {new }}\right)$ for six landmarks in a 3D MR image. $e_{\text {differential }}$ and $e_{\text {new }}$ denote the Euclidean distances to the reference positions, which were determined manually.

False Detections One problem with differential approaches is that often more than one landmark candidate is detected, i.e., we have to ensure that a correct candidate is selected for model initialization. To this end, we studied the suitability of using the fitting results to automatically identify false detections. For each landmark from above, we used all detected candidates to determine a set of initial values for the model parameters: For the left and right frontal ventricular horn as well as for the right occipital horn in the MR image we obtained two candidates, while for the left (right) zygomatic bone in the MR image we obtained three (five) candidates. In the case of the other landmarks, only one correct candidate was detected. We then compared the fitting results obtained for each candidate based on the value of the fitting measure divided by the surface area (the normalization was done to avoid a bias due to the surface area). We found that in all cases but one, the selection of a correct candidate actually resulted in the best fitting result. For the right occipital horn, it turned out that the detected two candidates are both correct in the sense that they refer to two prominent anatomical loci at the tip of the occipital horn.

\section{Conclusion}

In this paper, we introduced a new approach to 3D landmark localization based on deformable geometric models. By fitting these models to the surface at the landmark at hand, we obtain accurate estimates of the 3D landmark positions. Experimental results using 3D MR and CT images demonstrated the applicability of our approach. The utilization of differential approaches for determining initial values for the model parameters resulted in reasonable initializations. Using manually determined positions as ground truth, we analyzed in detail the accuracy of the estimated 3D landmark positions obtained from the fitting results. In particular, we compared the localization results with those of a differential approach. It turned out that our new approach based on deformable models significantly improves the localization accuracy and also reduces the number of false detections. Future work will include further experiments using clinical data. Also, extensions of the geometric models will be studied to capture a broader range of shapes. 


\section{References}

[1] E. Bardinet, L.D. Cohen, and N. Ayache. Superquadrics and Free-Form Deformations: A Global Model to Fit and Track 3D Medical Data. In N. Ayache, ed., Proc. CVRMed'95, LNCS 905, pp. 319-326. Springer-Verlag, Berlin, 1995.

[2] R. Bertolini and G. Leutert. Atlas der Anatomie des Menschen. Band 3: Kopf, Hals, Gehirn, Rückenmark und Sinnesorgane. Springer-Verlag, Berlin, 1982.

[3] K. Delibasis and P.E. Undrill. Anatomical object recognition using deformable geometric models. Image and Vision Computing, 12(7):423-433, 1994.

[4] L.M.J. Florack, B.M. ter Romeny, J.J. Koenderink, and M.A. Viergever. General Intensity Transformations and Differential Invariants. Journal of Mathematical Imaging and Vision, 4(2):171-187, 1994.

[5] L. Floreby, L. Sönmo, and K. Sjögreen. Boundary Finding Using Fourier Surfaces of Increasing Order. In A.K. Jain, S. Venkatesh, and B.C. Lovell, eds., Proc. ICPR'98, pp. 465-467. IEEE Computer Society Press, Los Alamitos, CA, 1998.

[6] S. Frantz, K. Rohr, and H.S. Stiehl. Improving the Detection Performance in Semi-automatic Landmark Extraction. In C.J. Taylor and A.C.F. Colchester, eds., Proc. MICCAI'99, LNCS 1679, pp. 253-262. Springer-Verlag, Berlin, 1999.

[7] A. Kelemen, G. Székely, and G. Gerig. Three-dimensional Model-based Segmentation of Brain MRI. In B. Vemuri, ed., Proc. IEEE Workshop on Biomedical Image Analysis, pp. 4-13. IEEE Computer Society Press, Los Alamitos, CA, 1998.

[8] T. McInerney and D. Terzopoulos. Deformable Models in Medical Image Analysis: A Survey. Medical Image Analysis, 1(2):91-108, 1996.

[9] E.H.W. Meijering, K.J. Zuiderveld, and M.A. Viergever. Image Reconstruction by Convolution with Symmetrical Piecewise nth-Order Polynomial Kernels. IEEE Trans. on Image Processing, 8(2):192-201, 1999.

[10] W.H. Press, B.P. Flannery, S.A. Teukolsky, and W.T. Vetterling. Numerical Recipes in C. Cambridge University Press, 1988.

[11] K. Rohr. On 3D differential operators for detecting point landmarks. Image and Vision Computing, 15(3):219-233, 1997.

[12] J. Sobotta. Atlas der Anatomie des Menschen. Band 1: Kopf, Hals, obere Extremität, Haut. Urban \& Schwarzenberg, München, 19th edition, 1988.

[13] F. Solina and R. Bajcsy. Recovery of Parametric Models from Range Images: the Case for Superquadrics with Global Deformations. IEEE Trans. on Pattern Analysis and Machine Intelligence, 12(2):131-147, 1990.

[14] L.H. Staib and J.S. Duncan. Model-based Deformable Surface Finding for Medical Images. IEEE Trans. on Medical Imaging, 15(5):720-730, 1996.

[15] D. Terzopoulos, A. Witkin, and M. Kass. Constraints on deformable models: recovering 3D shape and nonrigid motion. Artificial Intelligence, 36(1):91-123, 1988.

[16] D. Terzopoulos and D. Metaxas. Dynamic 3D Models with Local and Global Deformations: Deformable Superquadrics. IEEE Trans. on Pattern Analysis and Machine Intelligence, 13(7):703-714, 1991.

[17] J.-P. Thirion. New Feature Points based on Geometric Invariants for 3D Image Registration. Internat. Journal of Computer Vision, 18(2):121-137, 1996.

[18] M. Unser, A. Aldroubi, and M. Eden. B-Spline Signal Processing: Part I-Theory. IEEE Trans. on Signal Processing, 41(2):821-833, 1993.

[19] B.C. Vemuri and A. Radisavljevic. Multiresolution Stochastic Hybrid Shape Models with Fractal Priors. ACM Trans. on Graphics, 13(2):177-207, 1994.

[20] M. Worring, A.W.M. Smeulders, L.H. Staib, and J.S. Duncan. Parameterized feasible boundaries in gradient vector fields. In H.H. Barrett and A.F. Gmitro, eds., Proc. IPMI'93, LNCS 687, pp. 48-61. Springer-Verlag, Berlin, 1993. 